Characterizing optimality among three-decision procedures for directional conclusions

Fredrik Jonsson 



\title{
Characterizing optimality among three-decision procedures for directional conclusions
}

\author{
Fredrik Jonsson, Uppsala University
}

\begin{abstract}
Jones and Tukey [4] proposed three-decision procedures for directional conclusions in statistical inference, considered as an alternative to the conventional usage of one- and two-tailed significance testing. Morever, they suggested considering a procedure to be optimal in case indefinite results were minimized among all procedures with a given control of error. First, we argue by example that this characterization of optimality is not very fruitful when interpreted in the strong sense of uniform minimization. Next, imposing a further regularity condition on the comparative class of procedures, we relate the suggested characterization to optimality criteria from test theory (UMP unbiasedness). Similarly, we also consider characterizing optimality in terms of maximizing correct decision rates and minimizing incorrect decision rates. Finally, we demonstrate the applicability of the three considered characterizations with respect to exponential families of distributions.
\end{abstract}

\section{Introduction}

Lyle Jones and John Tukey [4] considered significance testing in the light of twosample mean-comparisons (with population means $\mu_{A}$ and $\mu_{B}$ ). They proposed replacing conventional two-sided $t$-testing of the hypothesis $H_{0}: \mu_{A}=\mu_{B}$ with procedures analyzing data in terms of one of three conclusions: "(a) act as if $\mu_{A}>\mu_{B}$; (b) act as if $\mu_{A}<\mu_{B} ;$ (c) act as if the sign of $\mu_{A}-\mu_{B}$ is indefinite, or not (yet) determined" ([4, p. 412]). Moreover, they suggested the perspective that

[...] a conclusion is in error only when it is "a reversal", when it asserts one direction while the (unknown) truth is the other direction [4, p. 412].

Their procedure is known, in conventional terminology, as simultaneous level- $\alpha$ onesided $t$-testing of both directions, with conclusions (a) and (b) if the corresponding opposite direction is rejected, and conclusion (c) if none of the two $p$-values falls below the given level $\alpha$.

Note that, if the true population means are essentially equal, then the correct and the incorrect direction are both inferred with rate $\alpha$ with the above procedure. Hence, definite conclusions are then obtained at rate $2 \alpha$. With traditional perspectives,

AMS 2000 subject classifications. Primary 62F03, 62A01; Secondary 62C99.

Keywords and phrases. Significance testing, directional inference, multiple decision procedures, simultaneous inference, optimality.

Date. November 15, 2011 
definite conclusions, or "significant changes", reject the possibility of true changes that are vanishingly small on the given scale of measurement (cf. [9, p. 356]). Thus, simultaneous level- $\alpha$ one-sided testing is traditionally considered to be essentially equivalent to level- $2 \alpha$ two-sided testing in this context, with "error level" $2 \alpha$.

Regarding optimality, a statistical test is said to be uniformly most powerful (UMP) if non-rejection rates, at situations where the null hypothesis is false, are minimized among procedures with controlled false rejection rates. For the three-decision setting, Jones and Tukey suggested the similar perspective:

We want to control the rate of error, the reversal rate, while minimizing wasted opportunity, that is, while minimizing indefinite results $([4, \mathrm{p}$. 412]).

In test theory, minimizing indefinite decision rates (non-rejections) on the alternative hypothesis is equivalent to maximizing correct decision rates (power), since only two possible decisions are available. However, maximizing rates of correct directional conclusion is not equivalent to minimizing rates of indefinite conclusions in the threedecision setting (cf. Section 5).

In the following we formulate a general framework for three-decision procedures (Section 2). We then consider three characterizations of optimality:

- Maximized rates of correct conclusion;

- Minimized rates of indefinite conclusion;

- Minimized rates of incorrect conclusion.

Moreover, we relate the three perspectives to well-known criteria from test theory (Sections 4-6). Finally, we consider the applicability of the three perspectives with respect to directional inferences for multi-parameter exponential families (Section 7).

\subsection{The a priori unlikelihood that the null hypothesis is true}

Jones and Tukey commented, as a critique of two-sided $t$-testing, that

[...] point hypotheses about parameters in real-world populations that are subjected to different treatments, are always untrue when calculations are carried to enough decimal places [4, p. 411].

A similar statement is given by Tukey in [11]:

All we know about the world teaches us that the effects of A and B are always different - in some decimal place - for any A and B [11, p. 100].

Hence, it is argued in [4], that the new proposal is more appropriate, since it "avoids the unrealistic postulation of a null hypothesis" ([4, p. 411]).

It may thus seem natural, regarding mathematical formulations of the proposal, to exclude the null hypothesis from the statistical model. For instance, Juliet Shaffer summarizes the Tukey-Jones directional proposal as follows,

[...] the usual 2-sided, equal-tails null hypothesis test at level $\alpha$ can be reinterpreted as simultaneous tests of 2 directional inequality hypotheses, each at level $\alpha / 2[\ldots]$ the maximum propability of a Type I error is $\alpha / 2$ if the truth of the null hypothesis is considered impossible ([9, p. 356]). 
Similarly, Shaffer refers in $[10$, p. 13] to the proposal in terms of considering null hypotheses impossible, and consequently, in terms of excluding corresponding parameter values from statistical models.

Moreover, it seems implicit from Shaffer's comment [9], that application of the Tukey-Jones directional proposal requires belief in the impossibility of the null hypothesis. It was also suggested that,

[...] not everyone may agree on the impossibility of the null hypothesis

$[\ldots]([9$, p. 357]).

Regarding the setting of mathematical models introduced in Section 2, no precise state of affairs is excluded on the grounds of "being impossible in practice". On the other hand, where the traditional null hypothesis is considered as a specific phenomenon (of "no effect"), it now instead occurs as a boundary state of affairs with respect to two possible directions.

Confer Nickerson [8, p. 263] for a review of related discussions (and a review of common missunderstandings) regarding the current practice of significance testing. Confer also Goudey [3], discussing the relevance of applying the Tukey-Jones directional proposal in environmental statistics.

\section{Uniformly optimal three-decision procedures}

Regarding the previously mentioned example from [4], consider the associated statistical model of normal distributions. We suggest dividing its parameter space into two regions specified by:

$$
H_{1}: \mu_{A} \leq \mu_{B}, \quad H_{2}: \mu_{A}>\mu_{B} .
$$

A corresponding three-decision procedure concludes either: (a) in favor of $H_{1}$; (b) in favor of $\mathrm{H}_{2}$; or (c) no definite decision.

Including the parameter value $\mu_{A}=\mu_{B}$ within $H_{1}$ above might be regarded as an act of convention. In fact, $H_{1}$ may be considered as a mirrored copy of $H_{2}$. Note that the distributional behaviour, regarding the elements of the considered model, changes continuously with the parameters of the model. Thus, $\mu_{A}=\mu_{B}$ does not formally refer to an isolated phenomenon. On the other hand, $\mu_{A} \approx \mu_{B}$ (with respect to the given scale of measurement) typically refers to situations where it is highly uncertain whether $H_{1}$ or $H_{2}$ is true.

We consider extending the above setting to a general formulation, with threedecision procedures on statistical models divided into two hypotheses. Thus, consider a random element $X$ and a set of probability distributions $\left\{P_{\theta}: \theta \in \Theta\right\}$, where each element is a possible law of $X$ (cf. [6, Section 1.1] and [7, Chapter 1]). Moreover, $\Theta$ is assumed to be partitioned into two disjoint subsets $\Theta_{1}$ and $\Theta_{2}$ (cf. [6, Section 3.1]).

Let $\left\{d_{1}, d_{2}, d_{3}\right\}$ refer to three possible decisions (cf. e.g. [2, Chapter 6$]$ for multiple decisions in decision theory). A corresponding randomized multiple decision rule (cf. [2, Chapter 6]) can then be represented by a function $\phi:\left(\phi_{1}, \phi_{2}, \phi_{3}\right)$. Here, each component is a nonnegative measurable function of $x$, with $\phi_{1}+\phi_{2}+\phi_{3} \equiv 1$.

Note (cf. [2, Section 1.5]) that $\phi$ transfers into a multiple decision rule $\delta_{\phi}$ (a function into the set $\left.\left\{d_{1}, d_{2}, d_{3}\right\}\right)$ through an independent random variable $Y$, uniformly 
distributed on the interval $[0,1]$,

$$
\delta_{\phi}(x, y)= \begin{cases}d_{1}, & \text { for } \quad y \leq \phi_{1}(x) \\ d_{2}, & \text { for } \quad \phi_{1}(x)<y \leq \phi_{1}(x)+\phi_{2}(x), \\ d_{3}, & \text { for } \quad \phi_{1}(x)+\phi_{2}(x)<y\end{cases}
$$

\subsection{Power, reversal and indefinite decision rates}

In the given setting with a partioning $\left\{\Theta_{1}, \Theta_{2}\right\}$ of $\Theta$ and three possible decisions $\left\{d_{1}, d_{2}, d_{3}\right\}$ we now associate $d_{1}$ with a conclusion $\theta \in \Theta_{1}, d_{2}$ with a conclusion $\theta \in \Theta_{2}$, and consider $d_{3}$ as an indefinite decision (a non-significant result). In the randomized setting thus consider, as functions on the parameter space and the set of possible three-decision rules,

$$
\begin{aligned}
& \pi(\theta, \phi)=\mathrm{E}_{\theta}\left(\phi_{1} I\left\{\theta \in \Theta_{1}\right\}+\phi_{2} I\left\{\theta \in \Theta_{2}\right\}\right), \quad \text { for } \theta \in \Theta \text {, } \\
& \rho(\theta, \phi)=\mathrm{E}_{\theta}\left(\phi_{2} I\left\{\theta \in \Theta_{1}\right\}+\phi_{1} I\left\{\theta \in \Theta_{2}\right\}\right), \quad \text { for } \theta \in \Theta \text {. }
\end{aligned}
$$

In other words, following the terminology of Jones and Tukey, for a given parameter $\theta$, consider $\rho$ as the corresponding "reversal rate", and $\pi$ as the rate of correct inference (or "power", with terminology borrowed from the setting of statistical testing). Note also that

$$
1-\pi-\rho=\mathrm{E}_{\theta}\left(\phi_{3}\right),
$$

refers to to the probability of a non-significant result (the "indefinite decision rate").

\subsection{Three-decision procedures and simultaneous testing}

A three-decision procedure $\phi$ may be regarded as a simultaneous test of $\Theta_{1}$ vs. $\Theta_{2}$, and $\Theta_{2}$ vs. $\Theta_{1}$, with corresponding test functions $\phi_{2}$ and $\phi_{1}$, respectively. Indeed, concluding $\theta \in \Theta_{1}$ can be interpreted as rejecting $\Theta_{2}$, and vice versa. Notably, controlling the reversal rates is equivalent to controlling the two levels of significance. Moreover, the power rate $\pi(\theta, \phi)$ of $\phi$ at $\theta$ is equal to the corresponding power rate of $\phi_{i}$, with $i=1,2$ depending on whether $\theta \in \Theta_{1}$ or $\theta \in \Theta_{2}$.

A characteristic feature in the above setting of three-decision procedures is thus that

$$
\phi_{1}+\phi_{2} \leq 1
$$

meaning that "colliding significances", or simultaneous rejections of both $\Theta_{1}$ and $\Theta_{2}$, are excluded. The class of three-decision procedures can be thus considered, for a given statistical model and partitioning $\left\{\Theta_{1}, \Theta_{2}\right\}$, as the corresponding subclass of simultaneous test procedures.

\subsection{Uniform optimality}

We adopt the following decision theoretic perspective on optimality: a given threedecision procedure $\phi^{*}$ is said to be uniformly optimal with respect to $D$ and $L$, where 
$D$ is a class of randomized three-decision procedures containing $\phi^{*}$, and $L$ is a loss function

$$
L: \Theta \times\left\{d_{1}, d_{2}, d_{3}\right\} \rightarrow \mathbb{R}_{\geq 0}
$$

in case expected loss is uniformly minimal, according to

$$
\mathrm{E}_{\theta}\left(L\left(\theta, \delta_{\phi^{*}}\right)\right) \leq \mathrm{E}_{\theta}\left(L\left(\theta, \delta_{\phi}\right), \quad \text { for any } \quad \theta \in \Theta \text { and } \phi \in D .\right.
$$

Note that the three perspectives on optimality in Section 1 correspond to the following three loss functions (respectively),

- $L_{1}\left(\theta, d_{i}\right)=1-I\left\{\theta \in \Theta_{1}\right\} I\{i=1\}-I\left\{\theta \in \Theta_{2}\right\} I\{i=2\}$; (everything but a correct decision is considered as a loss)

- $L_{2}\left(\theta, d_{i}\right)=I\{i=3\}$; (indefinite decisions are considered as losses)

- $L_{3}\left(\theta, d_{i}\right)=I\left\{\theta \in \Theta_{1}\right\} I\{i=2\}+I\left\{\theta \in \Theta_{2}\right\} I\{i=1\}$. (incorrect decisions are considered as losses)

Note also that, in the above randomized perspective on three-decision procedures $\phi:\left(\phi_{1}, \phi_{2}, \phi_{3}\right)$, with the notions of power and reversal rates from Section 2.1 , the expected loss is given by (respectively),

- $\mathrm{E}_{\theta}\left(L_{1}\left(\theta, \delta_{\phi}\right)=1-\pi(\theta, \phi)\right.$;

- $\mathrm{E}_{\theta}\left(L_{2}\left(\theta, \delta_{\phi}\right)=1-\pi(\theta, \phi)-\rho(\theta, \phi)\right.$;

- $\mathrm{E}_{\theta}\left(L_{3}\left(\theta, \delta_{\phi}\right)=\rho(\theta, \phi)\right.$;

Finally, for a given class of procedures $D$, note that optimality with respect to both $L_{2}$ and $L_{3}$ implies optimality with respect to $L_{1}$. Indeed, combining

$$
\pi\left(\theta, \phi^{*}\right)+\rho\left(\theta, \phi^{*}\right) \geq \pi(\theta, \phi)+\rho(\theta, \phi),
$$

with

$$
\rho\left(\theta, \phi^{*}\right) \leq \rho(\theta, \phi)
$$

implies

$$
\pi\left(\theta, \phi^{*}\right) \geq \pi(\theta, \phi)+\rho(\theta, \phi)-\rho\left(\theta, \phi^{*}\right) \geq \pi(\theta, \phi) .
$$

\section{A counterexample}

Recall the Tukey-Jones suggestion of characterizing optimality as minimized wasted opportunity under control of error. Thus, consider uniform optimality with respect to $L_{2}$ from Section 2.3, and the following class of three-decision procedures:

$$
D=\{\phi: \rho(\theta, \phi) \leq \alpha, \quad \text { for } \quad \theta \in \Theta\} .
$$


As a simple example, consider the statistical model $\{\mathrm{N}(\mu, 1): \mu \in \mathbb{R}\}$ of unit variance normal distributions, with parameter space divided into

$$
H_{1}: \mu \leq 0, \quad H_{2}: \mu>0 .
$$

A natural three-decision procedure $\phi^{*}:\left(\phi_{1}^{*}, \phi_{2}^{*}, \phi_{3}^{*}\right)$, corresponding to " $z$-testing", is then given by (for a given $\alpha \leq 1 / 2$ ),

$$
\begin{aligned}
\phi_{1}^{*}(x) & =I\left\{x<-z_{\alpha}\right\}, \\
\phi_{2}^{*}(x) & =I\left\{x>z_{\alpha}\right\}, \\
\phi_{3}^{*}(x) & =I\left\{-z_{\alpha} \leq x \leq z_{\alpha}\right\},
\end{aligned}
$$

with $z_{\alpha}=\Phi^{-1}(1-\alpha)$, with respect to the standard normal distribution function $\Phi$. Here, indefinite decision rates are given by

$$
\mathrm{E}_{\mu}\left(\phi_{3}^{*}\right)=\Phi\left(z_{\alpha}-\mu\right)-\Phi\left(-z_{\alpha}-\mu\right),
$$

and reversal rates are given by

$$
\rho\left(\mu, \phi^{*}\right)=\Phi\left(-z_{\alpha}-\mu\right) I\{\mu>0\}+\Phi\left(-z_{\alpha}+\mu\right) I\{\mu \leq 0\}=\Phi\left(-z_{\alpha}-|\mu|\right) \leq \alpha .
$$

Now, compare $\phi^{*}$ with $\widetilde{\phi}:\left(\widetilde{\phi}_{1}, \widetilde{\phi}_{2}, \widetilde{\phi}_{3}\right)$ given by

$$
\begin{aligned}
& \widetilde{\phi}_{1}(x)=\alpha, \\
& \widetilde{\phi}_{2}(x)=(1-\alpha) I\left\{x>z_{\alpha /(1-\alpha)}\right\}, \\
& \widetilde{\phi}_{3}(x)=(1-\alpha) I\left\{x \leq z_{\alpha /(1-\alpha)}\right\} .
\end{aligned}
$$

Here, indefinite decision rates are given by

$$
\mathrm{E}_{\mu}\left(\widetilde{\phi}_{3}\right)=(1-\alpha) \Phi\left(z_{\alpha /(1-\alpha)}-\mu\right),
$$

and reversal rates are given by

$$
\begin{aligned}
\rho(\mu, \widetilde{\phi}) & =\alpha I\{\mu>0\}+(1-\alpha) \Phi\left(-z_{\alpha /(1-\alpha)}+\mu\right) I\{\mu \leq 0\} \\
& \leq \alpha I\{\mu>0\}+(1-\alpha) \Phi\left(-z_{\alpha /(1-\alpha)}\right) I\{\mu \leq 0\}=\alpha .
\end{aligned}
$$

Consider the difference between the indefinite decision rates (2) and the indefinite decision rates (1), as a function of $\mu$,

$$
\Delta(\mu)=(1-\alpha) \Phi\left(z_{\alpha /(1-\alpha)}-\mu\right)+\Phi\left(-z_{\alpha}-\mu\right)-\Phi\left(z_{\alpha}-\mu\right) .
$$

To begin with,

$$
\begin{aligned}
\Delta(0) & =(1-\alpha) \Phi\left(z_{\alpha /(1-\alpha)}\right)+\Phi\left(-z_{\alpha}\right)-\Phi\left(z_{\alpha}\right) \\
& =(1-\alpha)(1-\alpha /(1-\alpha))+\alpha-(1-\alpha) \\
& =(1-\alpha)-\alpha+\alpha-(1-\alpha)=0 .
\end{aligned}
$$

Moreover, differentiation of $\Delta$ yields, with $\varphi$ referring to the standard normal density function,

$$
\begin{aligned}
\Delta^{\prime}(0) & =-(1-\alpha) \varphi\left(-z_{\alpha /(1-\alpha)}\right)-\varphi\left(-z_{\alpha}\right)+\varphi\left(z_{\alpha}\right) \\
& =-(1-\alpha) \varphi\left(-z_{\alpha /(1-\alpha)}\right)<0 .
\end{aligned}
$$

Thus, it cannot be the case that $\Delta(\mu) \geq 0$, for all $\mu$. To conclude, the indefinite decision rates of $\phi$ are not uniformly smaller than the corresponding indefinite decision rates of $\widetilde{\phi}$. 


\section{Maximizing power rates}

We begin from the perspective of identifying a three-decision procedure $\phi$ as a simultaneous test of $\Theta_{1}$ and $\Theta_{2}$, transferring optimality characterizations regarding the individual test procedures to the three-decision setting.

Recall that a uniformly most powerful (UMP) level- $\alpha$ test of a hypothesis $H$ maximizes power uniformly among all level- $\alpha$ tests of $H$. However, there are relatively few interesting statistical models in which UMP level- $\alpha$ tests exist. It is often the case that biased solutions can be constructed, in the sense that power may be maximized locally at the cost of a very poor global behaviour. Thus, Theorem 4.1 refers to the weaker form of "UMP unbiased optimality" of the two testing functions (cf. [6, Chapter 4]), which is a more commonly satisfied property in statistical models, referring to optimality among procedures with power exceeding the level of significance.

For the notion of uniform optimality with respect to a class of procedures $D$ and a loss function $L$, confer Section 2.3. Moreover, regarding the notions of power and reversal rates $\pi$ and $\rho$, confer Section 2.1.

Theorem 4.1. Let a statistical model $\left\{P_{\theta}: \theta \in \Theta\right\}$ and a partitioning $\left\{\Theta_{1}, \Theta_{2}\right\}$ of $\Theta$ be given. For any $\alpha$, a three-decision procedure $\phi^{*}$ satisfying conditions (i) and (ii) is uniformly optimal with respect to $D$ and $L$,

$$
\begin{aligned}
D & =\{\phi: \quad \rho(\theta, \phi) \leq \alpha, \pi(\theta, \phi) \geq \alpha, \text { for all } \theta \in \Theta .\}, \\
L\left(\theta, d_{i}\right) & =1-I\left\{\theta \in \Theta_{1}\right\} I\{i=1\}-I\left\{\theta \in \Theta_{2}\right\} I\{i=2\},
\end{aligned}
$$

(i) $\phi_{1}^{*}$ is UMP unbiased level- $\alpha$ for testing $\Theta_{2}$;

(ii) $\phi_{2}^{*}$ is UMP unbiased level- $\alpha$ for testing $\Theta_{1}$.

Proof. Let $\phi^{*}$ and $\phi$ be given, with $\phi^{*}$ satisfying conditions (i), (ii) and $\phi \in D$. It follows from the definition of $D$ that $\phi_{1}$ is unbiased level- $\alpha$ for testing $\Theta_{2}$. Indeed, the power function of $\phi_{1}$ is given by

$$
\mathrm{E}_{\theta}\left(\phi_{1}\right)=\rho(\theta, \phi) I\left\{\theta \in \Theta_{2}\right\}+\pi(\theta, \phi) I\left\{\theta \in \Theta_{1}\right\} .
$$

Similarly, it also follows that $\phi_{2}$ is unbiased level- $\alpha$ for testing $\Theta_{1}$, noting that the power function of $\phi_{2}$ is given by

$$
\mathrm{E}_{\theta}\left(\phi_{2}\right)=\rho(\theta, \phi) I\left\{\theta \in \Theta_{1}\right\}+\pi(\theta, \phi) I\left\{\theta \in \Theta_{2}\right\} .
$$

Thus, $\phi_{1}^{*}$ and $\phi_{2}^{*}$ are more powerful compared to $\phi_{1}$ and $\phi_{2}$, due to assumptions (i) and (ii), or in other words, $\pi\left(\theta, \phi^{*}\right) \geq \pi(\theta, \phi)$, for all $\theta \in \Theta$.

\section{Minimizing indefinite decision rates}

Probabilities of indefinite decisions are not necessarily minimized by an optimal procedure in the sense of Theorem 4.1. As an example, consider the situation in Section 3. Here, $\phi^{*}$ satisifies conditions (i) and (ii) in Theorem 4.1. Also, the comparative procedure $\widetilde{\phi}$ belongs to the corresponding class $D$. Indeed, its power rate is given by

$$
\begin{aligned}
\pi(\mu, \widetilde{\phi}) & =\alpha I\{\mu \leq 0\}+(1-\alpha) \Phi\left(-z_{\alpha /(1-\alpha)}+\mu\right) I\{\mu>0\} \\
& \geq \alpha I\{\mu \leq 0\}+(1-\alpha) \Phi\left(-z_{\alpha /(1-\alpha)}\right) I\{\mu>0\}=\alpha
\end{aligned}
$$


We noted in Section 3 that the indefinite decision rates of $\widetilde{\phi}$ were not uniformly smaller than the indefinite decision rates of $\phi^{*}$.

However, $\phi^{*}$ in Section 3 minimizes indefinite decision rates within a less general class of procedures, according to the following result (Theorem 5.1). Here, we impose on the comparative class $D$ that indefinite decision rates should not exceed $1-2 \alpha$. Hence, uniform minimization of indefinite decision rates is associated with optimal rejection of the boundary between the two hypotheses. For simplicity, attention is restricted to euclidean parameter spaces with a continuous behaviour, according to the assumption that

$$
\theta \mapsto \mathrm{E}_{\theta} \varphi
$$

is a continuous function, for any test function $\varphi$. Confer [6, Section 2.7] for a proof of this behaviour regarding exponential families of distributions.

Theorem 5.1. Let a statistical model $\left\{P_{\theta}: \theta \in \Theta\right\}$, for a subset $\Theta \subset \mathbb{R}^{d}$, and a corresponding partitioning $\left\{\Theta_{1}, \Theta_{2}\right\}$ of $\Theta$ be given. Moreover, let $\Theta_{0}$ refer to the boundary of $\Theta_{1}$, as a subset of $\Theta$. Assume that $\theta \mapsto \mathrm{E}_{\theta} \varphi$ is continuous on $\Theta_{0}$, for any function $\varphi$. For any $\alpha$, a three-decision procedure $\phi^{*}$ satisfying condition $(i)$ is uniformly optimal with respect to $D$ and $L$,

$$
\begin{aligned}
D & =\{\phi: \rho(\theta, \phi) \leq \alpha, \pi(\theta, \phi)+\rho(\theta, \phi) \geq 2 \alpha, \text { for all } \theta \in \Theta .\}, \\
L\left(\theta, d_{i}\right) & =I\{i=3\},
\end{aligned}
$$

(i) $\phi_{1}^{*}+\phi_{2}^{*}$ is UMP unbiased level-2 $\alpha$ for testing $\Theta_{0}$.

Proof. Let $\phi^{*}$ and $\phi$ be given, with $\phi^{*}$ satisfying condition (i) and $\phi \in D$. Concerning $\phi$, it follows from the definition of $D$ and the assumption of continuity that

$$
\pi(\theta, \phi)+\rho(\theta, \phi)=2 \alpha, \text { for } \theta \in \Theta_{0} .
$$

Indeed, for a given $\theta \in \Theta_{0}$, let convergent sequences $\left\{\theta_{1, n}\right\}_{n \geq 1}$ and $\left\{\theta_{2, n}\right\}_{n \geq 1}$ be given, with $\theta_{i, n} \in \Theta_{i}$. Thus,

$$
\mathrm{E}_{\theta_{1, n}}\left(\phi_{2}\right) \leq \alpha, \quad \mathrm{E}_{\theta_{2, n}}\left(\phi_{1}\right) \leq \alpha,
$$

and by continuity,

$$
\pi(\theta, \phi)+\rho(\theta, \phi)=\mathrm{E}_{\theta}\left(\phi_{2}+\phi_{1}\right) \leq 2 \alpha,
$$

which proves (3). Due to (3), $\phi_{1}+\phi_{2}$ is unbiased level-2 $\alpha$ for testing $\Theta_{0}$. Hence, $\phi_{1}^{*}+\phi_{2}^{*}$ is more powerful than $\phi_{1}+\phi_{2}$ as a test of $\Theta_{0}$, due to assumption (i). In other words,

$$
\pi\left(\theta, \phi^{*}\right)+\rho\left(\theta, \phi^{*}\right) \geq \pi(\theta, \phi)+\rho(\theta, \phi), \quad \text { for } \quad \theta \notin \Theta_{0} .
$$

Also, by continuity and assumption (i),

$$
\pi\left(\theta, \phi^{*}\right)+\rho\left(\theta, \phi^{*}\right)=2 \alpha, \quad \text { for } \quad \theta \in \Theta_{0} .
$$

Combining (3)-(5) we obtain

$$
\pi\left(\theta, \phi^{*}\right)+\rho\left(\theta, \phi^{*}\right) \geq \pi(\theta, \phi)+\rho(\theta, \phi), \quad \text { for all } \quad \theta \in \Theta,
$$

which proves that expected loss is uniformly minimized. 


\section{$6 \quad$ Minimizing reversal rates}

Theorem 6.1 is the analogue of Theorem 4.1, with maximization of correct decision rates replaced by minimization of incorrect decision rates.

Theorem 6.1. Let a statistical model $\left\{P_{\theta}: \theta \in \Theta\right\}$ and a partitioning $\left\{\Theta_{1}, \Theta_{2}\right\}$ be given. For any $\alpha$, a three-decision procedure $\phi^{*}$ satisfying conditions (i) and (ii) is uniformly optimal with respect to $D$ and $L$,

$$
\begin{aligned}
D & =\{\phi: \quad \rho(\theta, \phi) \leq \alpha, \pi(\theta, \phi) \geq \alpha, \text { for all } \theta \in \Theta .\} \\
L\left(\theta, d_{i}\right) & =I\left\{\theta \in \Theta_{1}\right\} I\{i=2\}+I\left\{\theta \in \Theta_{2}\right\} I\{i=1\}
\end{aligned}
$$

(i) $1-\phi_{1}^{*}$ is UMP unbiased level- $(1-\alpha)$ for testing $\Theta_{1}$;

(ii) $1-\phi_{2}^{*}$ is UMP unbiased level- $(1-\alpha)$ for testing $\Theta_{2}$.

Proof. Let $\phi^{*}$ and $\phi$ be given, with $\phi^{*}$ satisfying conditions (i) and (ii) and $\phi \in D$. Assuming $\theta \in \Theta_{1}, 1-\phi_{1}$ satisfies

$$
\mathrm{E}_{\theta}\left(1-\phi_{1}\right)=1-\pi(\theta, \phi) \leq 1-\alpha
$$

Similarly, given $\theta \in \Theta_{2}, 1-\phi_{1}$ satisfies

$$
\mathrm{E}_{\theta}\left(1-\phi_{1}\right)=1-\rho(\theta, \phi) \geq 1-\alpha .
$$

Thus, $1-\phi_{1}$ is unbiased level- $(1-\alpha)$ for testing $\Theta_{1}$. We hence deduce that, in view of condition (i), $1-\phi_{1}^{*}$ is more powerful for testing $\Theta_{1}$, i.e. that, given $\theta \in \Theta_{2}$,

$$
1-\rho(\theta, \phi)=\mathrm{E}_{\theta}\left(1-\phi_{1}\right) \leq \mathrm{E}_{\theta}\left(1-\phi_{1}^{*}\right)=1-\rho\left(\theta, \phi^{*}\right) .
$$

By symmetry, $1-\phi_{2}$ is unbiased level- $(1-\alpha)$ for testing $\Theta_{2}$. In view of condition (ii), we thus deduce that, given $\theta \in \Theta_{1}$,

$$
1-\rho(\theta, \phi)=\mathrm{E}_{\theta}\left(1-\phi_{2}\right) \leq \mathrm{E}_{\theta}\left(1-\phi_{2}^{*}\right)=1-\rho\left(\theta, \phi^{*}\right) .
$$

Combining (6)-(7) we conclude that

$$
\mathrm{E}_{\theta}\left(L\left(\theta, \delta_{\phi^{*}}\right) \leq \mathrm{E}_{\theta}\left(L\left(\theta, \delta_{\phi}\right), \quad \text { for all } \theta \in \Theta\right.\right.
$$

completing the proof of the theorem.

\section{Exponential families of distributions}

Following the exposition of conditional statistical testing with respect to multiparameter exponential families given in [6, Section 4.4], we now consider a convex parameter space $\Theta \subset \mathbb{R}^{k+1}$, assuming that it is not contained in a linear subspace of dimension $<k+1$. The elements of $\Theta$ are referred to as $(\theta, \vartheta)$, with $\theta \in \mathbb{R}$ and $\vartheta \in \mathbb{R}^{k}$. Next, with respect to some probability measure on $\mathbb{R}^{k+1}$, consider a family of probability density functions of the following form:

$$
C(\theta, \vartheta) \exp \left(\theta U(u, t)+\sum_{i=1}^{k} \vartheta_{i} t_{i}\right), \quad(\theta, \vartheta) \in \Theta .
$$


Moreover, consider partitioning $\Theta$ into non-empty subsets $\Theta_{1}$ and $\Theta_{2}$ by

$$
\Theta_{1}: \theta \leq \theta_{0}, \quad \Theta_{2}: \theta>\theta_{0},
$$

for some $\theta_{0} \in \mathbb{R}$. Finally, given a maximal reversal rate $\alpha \leq 1 / 2$, consider real-valued functions $t \mapsto C_{i}(t), t \mapsto \gamma_{i}(t)$, for $i=1,2$ and $0 \leq \gamma_{i} \leq 1$ determined by:

$$
\begin{aligned}
& \phi_{1}^{*}(u, t)=I\left\{u<C_{1}(t)\right\}+\gamma_{1}(t) I\left\{u=C_{1}(t)\right\}, \\
& \phi_{2}^{*}(u, t)=I\left\{u>C_{2}(t)\right\}+\gamma_{2}(t) I\left\{u=C_{2}(t)\right\}, \\
& \phi_{3}^{*}(u, t)=I\left\{C_{1}(t)<u<C_{2}(t)\right\}+\sum_{i=1,2}\left(1-\gamma_{i}(t)\right) I\left\{u=C_{i}(t)\right\},
\end{aligned}
$$

and the equations:

$$
\begin{aligned}
\alpha & =\mathrm{E}_{\theta_{0}}\left(\phi_{2}^{*}(U, T) \mid T=t\right), \\
\alpha & =\mathrm{E}_{\theta_{0}}\left(\phi_{1}^{*}(U, T) \mid T=t\right) .
\end{aligned}
$$

We begin by applying Theorems 4.1 and 6.1 to $\phi^{*}:\left(\phi_{1}^{*}, \phi_{2}^{*}, \phi_{3}^{*}\right)$, referring to $[6$, Theorem 4.4.1] for the verification of the relevant conditions (i) and (ii) in Theorems 4.1 and 6.1 .

Corollary 7.1. The three-decision procedure $\phi^{*}$ defined by (10)-(14) maximizes power rates uniformly and minimizes reversal rates uniformly among procedures with reversal rates bounded by $\alpha$ (from above) and power rates bounded by $\alpha$ (from below).

Next, regarding the relevance of Theorem 5.1, condition (i) transfers to a wellknown unbiasedness condition (15). Here, confer [6, Section 4.2].

Corollary 7.2. Assume that the three-decision procedure $\phi^{*}$ defined by (10)-(14) satisfies the following equations:

$$
\mathrm{E}_{\theta_{0}}\left(U\left(\phi_{1}^{*}(U, T)+\phi_{2}^{*}(U, T)\right) \mid T=t\right)=2 \alpha \mathrm{E}_{\theta_{0}}(U \mid T=t) .
$$

Then, $\phi^{*}$ minimizes indefinite decision rates uniformly among procedures with reversal rates bounded by $\alpha$ (from above) and indefinite decision rates bounded by $1-2 \alpha$ (from above).

\section{Concluding remarks}

We note that (cf. [6, Section 4.2]) condition (15) is fulfilled if the conditional distribution of $U$ given $T=t$ is symmetric around some point, for all $t$. This is the case when comparing two normal population means, as in the example of Jones and Tukey, and in the similar comparison of Poisson means and binomial means with equal number of summands (that is, when assessing the ordering of the corresponding means). However, the condition is not fulfilled when assessing the unknown parameter $p$ regarding a binomial distribution, unless the division is made at $p=1 / 2$. Similarly, the condition is generally not fulfilled when comparing the means of two binomial distributions with unequal number of summands.

Finally, one may also note that Bahadur [1] proved an optimality result regarding two-sample mean-comparisons which is closely related to the present context of threedecision procedures and Corollaries 7.1-7.2. 


\section{Acknowledgment}

I would like to thank Silvelyn Zwanzig for valuable comments and suggestions regarding earlier versions of this paper.

\section{References}

[1] Bahadur, R.R. (1952). A property of the t-statistic. Sankhya 12, 79-88.

[2] Ferguson, T.S (1967). Mathematical Statistics: A Decision Theoretic Approach. Academic Press.

[3] Goudey, R. (2007). Do statistical inferences allowing three alternative decisions give better feedback for environmentally precautionary decision-making? J. Env. Man. 85, 338-344.

[4] Jones, L.V. And Tukey, J.W. (2000). A sensible formulation of the significance test. Psychol. Meth. 5, 411-414.

[5] Lehmann, E.L. (1957). A theory of some multiple decision problems, Part II. Ann. Math. Statist. 28, 547-572.

[6] Lehmann, E.L. and Romano, J.P. (2005). Testing Statistical Hypotheses. Third Edition. Springer-Verlag.

[7] Liese, F. And Miescke, K.-J. (2008). Statistical Decision Theory. SpringerVerlag.

[8] Nickerson, R.S. (2000). Null hypothesis significance testing: a review of an old and continuing controversy. Psychol. Meth. 5, 241-301.

[9] Shaffer, J.P. (2002). Multiplicity, directional (Type III) errors, and the null hypothesis. Psychol. Meth. 7, 356-369.

[10] Shaffer, J.P. (2004). Optimality results in multiple testing. The First Erich L. Lehmann Symposium - Optimality, Lecture Notes-Monograph Series, Vol. 44. Institute of Mathematical Statistics, 11-35.

[11] Tukey, J.W. (1991). The philosophy of multiple comparisons. Statist. Sci. 6, $100-116$. 